\title{
Study on the Classification and Expression of Chapter Heading and Antithesis of the Water Margin
}

\author{
Yu Deng \\ Yulin University, Yulin, Shaanxi, 719000
}

Keywords: Chapter heading, Antithesis, Zhanghui Novel, Water Margin

\begin{abstract}
The system of Zhanghui novel is an external structural style of the development of ancient Chinese novels. It is the representative feature of Zhanghui novels. Its appearance replaces the traditional novels based on the volume. "Water Margin" as the earliest in Chinese history written in the vernacular one of the novel, its chapter heading has important significance. This paper analyzes the return of the "Water Margin" from two aspects of form and content, and summarizes the rules of antithesis according to some signs. At the same time, this article also analyzes the effect of the expression.
\end{abstract}

\section{Introduction}

The development of the novel, in short, first experienced the early embryonic stage of the pre-Qin and Han dynasties. Then Ban Gu and Huan Tan have made a theoretical explanation for novel. Till the Wei and Jin Dynasties, the novel has a new development, due to social atmosphere and cultural beliefs and other factors, mystery novels began to prevail. To the Sui and Tang dynasties, the novel advanced towards a more mature stage, the classical short stories that were represented by legend began to rise, but this literary genre has not been developed of continuity. After the Song dynasty, it began to decline. On the other hand, the flourishing of the vernacular novels in the Song and Yuan Dynasties is different from the classical novels, which is a kind of new literature and art of the public class. There are also novels and lectures. The novels of Song and Yuan Dynasties can be regarded as a great change in ancient Chinese novels.

The novel is not a new literary genre in the development of Chinese classical literature. From the late Han Dynasty, there are note fictions, mystery novels, and anecdote, as well as ever-evolving genre of legendary literatures. In the song dynasty, it developed into a widely circulated story, and in the late Yuan dynasty, a more mature chapter was formed, and the Ming and Qing dynasties became the most prosperous period in the development of ancient Chinese novels. Among them, the most representative one is to replace the traditional volume with Chapter heading.

The system of Zhanghui novel is an external structural style of the development of ancient Chinese novels. Its basic characteristics are long, vernacular and return, that is, there is a pair of sentences as a title. Is the representative feature of the Zhanghui novel, from the early development of the single sentence title to the two sentences titles to the basic shape, double sentence titles became the main rhetorical way is antithesis. Therefore, the study of Zhanghui novels is not only the research category of classical literature, although its form is simple, but also related to the content of modern linguistics and rhetorics, and even contains ancient history, society, Culture and other research content.

"Water Margin" also known as "loyalty Water Margin", formerly known as "Biography of Heroes", the late Yuan and early Ming Dynasty works, is the first in Chinese history with the vernacular language written chapter novels. As an excellent literary works, "Water Margin" in the ideological and artistic creation has a great success can not be ignored, the development of the Zhanghui style to the novel also has a significant importance. 


\section{The Classification and Explanation of the Form of "Water Margin"}

The version this article uses is "Water Margin" with 120 chapter headings, this version in the system to maintain the tradition of the Song and Yuan Dynasties, draw lessons and the advantages of the Yuan and Ming dynasties. From the back of the head, to retain the words often appear in the form of words, such as "Zhang Tianshi pray plague plague, Hong Yuwei mischievous demon." Because this is a vernacular novel, the text is very close to the usual words, so the back of the form of the structure is relatively simple, but still continued neat, rigorous is the format. From the upper and lower sentence structure, the seven words and eight words mainly, which made a total of 54 words, accounting for $45 \%$ of the total, seven words a total of 59 , accounting for $49.1 \%$ of the total.

In addition, the "Water Margin" in the head appeared in the nine words and six pairs, although there are only two words, six words only five, but with the same period of other works have been significantly different. Such as the twenty-seventh back to the mother of the fork in the city of Mangzhou Road, the sale of meat Wudu head cross slope case Zhang Qing, seventy-six back Wu Jia Liang cloth four bucket five flag Song Gongming row Jiugong Bagua Zhen. Six words on the seventy-third round of the black whirlwind Qiao catch the ghosts Liang Shanbao twin, the first hundred and nine back to Wang Qing crossing the river was caught Song Jiang defeated Kou success, the ninth back to Li Kui dream Tian Tian Song Jiang Bing two points, Ninety four back to the victory down three will Li Kui reckless people, and the ninety-eight Zhang Qingyuan with Qiongying Wu Wu poison with the poison. Another major feature of the work is that the number of heads of the subject-predicate structure increases.

\section{According to the structure and form of Chapter Headings, it can be divided into the following categories}

\subsection{Subject-predicate structure}

This kind of chapter heading takes the largest proportion of a part of the 120 chapter heading, the subject of the structure of the heading accounted for more than half. Generally speaking, there are "three three three" structure, in which the verb some monosyllabic adverbs modified, and some are directly verbs. For example, the second back to the king of the head of the private private Yan'an County Jiulian long history of the history of the village, the third back to the history of Danglang night Huayin County Luti jurisdiction punched the town of Kansai, the sixth back to the nine-shaped long-Chi-chi-chuh, Cave Temple, the fourteenth back to the red hair ghost drunk lying Ling Guan Dian Chao Tian Wang Dongxi Village, the thirty-first back Zhang are blood flowers Diao Yuanyu House Wu Zuogu night walking centipede ridge, forty-sixth back to the disease Trouble Tsui Ping Shan desperately burning fire shop. There are "three one four" structure, the forty-second back to the village by the three volumes of the book Song Gongming met nine days mysterious woman, the thirty-seventh back to the rain in time will be the god of the black war of the black whirlwind.

Seven words for the "twenty-two three", such as the first sixteen back to Yang Chi escort gold and silver bear Wu with wisdom to take the first class, the twenty-second back Yanpo excuse Yuncheng County Zhu Tongyi Song Gongming, thirty-second Back to the martial arts drunk drilled bright brilliance song Song Jiang. Also see the "two one four" form, the fifty-ninth back to Wu with gold bell hanging Songjiang downtown Xiyue Huashan, sixty-third Song Jiang Bing hit the famous city wins wins Liangshan Park. Or "four three" structure, the forty-ninth back to the solution of the double-jailbreak Sun Li Sun Sun robbed robbery, there are the first hundred back Zhang Qingqiong British double construction Chen Guan Song Jiang with the Czech Republic.

\subsection{The subject-predicate structure with adverbial modifier of two or more words}

The beginning of the two nouns is followed by the form of a guest phrase, where one of the place nouns has an adverbial nature. Eight words have "five one two" form, such as the twentieth back to Liangshan Shanyi respect for the Chao Chai Yuncheng County on the moon to go Liu Tang, the 
forty back to Liangshan Park hero robbery court Bailongmiao hero small Yi Yi, thirty-sixth back Liangshan Bo Wu Yongzhuang Dai Zong Jieyang Ling Song Jiangfeng Li Jun, sixty-fourth back to the extension of the month of the month to make wins Song Gongming snow days to capture cable super. Or "sixty-two" form, such as the sixty back to the public Sun Shengmang Dangshan down the devil Chao Teng Wang Zengcheng City in the arrow, the first hundred and thirteen back to the chaos of the Mihu Long Taihuo Song Gongming Suzhou Conference Gai.

Seven words for the "May 2" structure, such as the twelfth back Liangshan Park forest washed off the grass Bianjing City Yangzhi selling knives, the first nine back to the forest washed large and fire Chao Chao Liangshan small boat, the twenty-third back to Henghai County Chai Jin Liu Jing Jin Gang Wu Song tiger, thirty-fifth back to the general hospital shop sent to the small Li Guang Liangshan shot wild, the ninth back to Wu Taishan Song Jiang Zen Chan Shuanglin Township Yanqing confused, the first one hundred and fourteen Back to the navy Song Jiang hanging filial piety Jinmen Zhang Shun to God, the first one hundred and ninety-nine back to Lu Zhishen Zhejiang Zuohua Song Komeito back home and so on.

The adverb of the adverb. Such as the eleventh back to Zhu Guishui Ting Shi Arrow Lin Chong snow at night Liangshan, the thirtieth return graceful three into the death row Wu Song Puyun Yunpu, the ninth back to the door of the door to the world, Two back to the firewood into the banquet Li Kui Yuan night trouble in Tokyo, the fifty-fourth back into the cloud dragon fighting method to break the high black whirlwind down the firewood, the first hundred and twenty back to Wang Qing for the rape Gong Du was beaten Military prisoners, the sixty-fifth back to the tower of the king of the dream of the holy waves in the white strip of water to report injustice, the first three hundred and thirty back to the management of the concubine brother died for the stage level for the cousin face, The key is to return to the purpose of the main purpose is to express the location, time, causality and other adverbs or positive phrases and verb relations.

With the phrase as the main body, that is, the content has two actions in parallel. Such as the fifteenth back to the school of Wu said that the three Ruan hit the public Gongsheng Sheng should be seven stars together, the fifty-sixth back to Wu with the time to steal a tattoo made Xu Ning up the mountain, the sixty-seventh back Songjiang tours horse step army Precipitation and fire two will be seventy times back no feather arrows flying heroes song Gongming abandoned grain capture warriors, the seventy-fifth back to live Lama robbed steal wine wine black tornado vowed imperial edict, the eighty-second back Liangshanpu sub-big to buy City Song Gongming all by the Zongan, the eighty-third Song Gongming Fengzhao breaking Da Liao Chen Qiao Yi tears cut off, the first hundred and sixty-six students talk laugh but the enemy is not a thorny city.

With a positive phrase chapter heading, mainly positive phrases plus cheong phrase. Such as the fifth back to the king of the bully sold into gold and silver monk peach blossom village, the thirteenth back to the vanguard of the East Guo Gong Gong green beast Beijing Duwu, forty-third back to the fake Li Kui cut the path of single black cyclone Yiling killed four Tiger and so on.

The form is in the "Water Margin" in the more rare, only the twenty-sixth back to stealing bone nine to send funeral for the head Wuwu sacrifice, the sixty-second replay Leng Jian Yan Qing rescue robbery court show Jumped, the twentieth vibration Zhenwei small Li Guangshen arrows to cover the county multi-star secret, the ninety-six Hui magic demon embarrassment Wu Longshan into the Yunlong soldiers Wai hundred Valley ridge, the first one hundred and one back to the grave insidious Produced by the chunk Chunyang flirtatious tragedy, the first hundred and eighty-eight Qiao Daqing clear fog take the city of small whirlwind possession of thieves, such as less than ten. This may be related to the characteristics of the Water Margin.

At the same time, in the upper and lower related to the head also has a similar to today's row than the right, such as the forty-seventh back fluttering carved two books of life and death book Song Gongming a fight wish Zhuang, forty-eight back To catch the king of short tiger song Gongming two fight Zhu Zhuangzhuang, the fifth fifty years to study the palm of your hand Shuang Kouming three dozen wish Chu Zhuang, which in the title of antithesis structure of the vertical formation of a multi-sentence. Another example is the seventy-eight back ten knots to take Liangshan Bo Song Gongming defeated the high lieutenant, the seventy-ninth back to Liu Tang fire war ship Song Jiang 
two defeated high lieutenant, eighty times Zhang Shun chisel leakage loach boat Song Jiang three defeated high Wei, is also a similar structure.

\section{Classification and Interpretation of the Contents of Chapter Headings in "Water Margin"}

From the content on the division, the antithesis of the title is relatively simple, mostly coordinate couplet and connected couplet.

The Chapter Heading with coordinate couplet, that is, the two people and the story is tied, "flower monk" and "leopard head" done things for the back of the two Coexistence of the plot, the logic is not strictly to undertake, progressive, causal and other relations. In addition to this, the eighth back to the forest leader with Cangzhou Road Lu Zhizhen wild wild boar forest, the twenty-eighth back to Wu Song Megumi peace of the village of benevolence wins happy forest, the thirty-third back Songjiang night to see the small Aoshan flowers Rongju Qingfengzhai , The thirty-fourth back to the town of three mountains of the Qingzhou Road, Thunderbolt fire night to go gravel field, forty-fourth back to the leopard leopard path every Daizong Guan Suo long street with Shi Xiu, fifty-third back to Dai Zong two take Gongsheng Sheng Li Kui independence Split the real people, the sixty-first back to Wu Zhizhi earn jade unicorn Zhang Shun night Jinsha crossing, the sixty-sixth back to the fire Cuiyun floor Wu Zongzhi take the name of the government, seventy-four back to Yan Qingzhi Pagoda Tian Tian Li Kui Shou Zhang Qiao sitting in the Ya, the eighty-fourth Song Gong Ming soldiers fight Jizhou City Lu Junyi war Yutian County, the first hundred and forty-four section of the section of the village to recruiting the new daughter-in-law cottage and the old strongman, the first hundred and ten back Yan Qingqiu Lin Wu Zhaoyan Song Jiang Dongjing City captive, the first one hundred and eighteen back to Lu Junyi war Yuling off Song Gongming wise to clear the cave and so on. From this expression, readers can feel the richness of the plot in a simple sentence.

The Chapter Heading with connected couplet, that is, between the next two there is causal, to undertake and progressive logical relationship. Such as the fifty-seventh back to Xu Ning teach the hook sickle song Songjiang big break ring horse, the fifty-fifth back to the high lieutenant Daxing three road soldiers call Yanzhu mercy ring horse, the sixty-ninth back to Dongping House mistaken Jiuge Long Song Gongming Release the two guns, the sixty-eight Song Gongming night hit the first city of Lu Junyi live Shi Wen Gong, the eighty-fifth Song Gongming night Yi Jin Guan Wu learn to learn from Wen'an County, the eighty-eighth back to the Yanjun array mixed days like Song Gongming Dream of the mysterious female law, the first one hundred and fifteen back to the soul of the soul of the soul of the Song Dynasty set Song Jiangzhi to take the navy and so on. This expression is mostly set up to highlight the relationship between the various characters in the course of the continuation of the plot, and to show a tense atmosphere to attract the reader.

\section{The Expression Effect of Chapter Heading in "Water Margin"}

Zhanghui novel is a narrative style, in each of which has covered a large number of storylines, back to the text as the outline of the outline of the outline of the summary, must be explained as clearly the main content of each back - people, even time and location. From the expression effect, the "Water Margin" back to the weakened the details of the description and the intense confrontation of the scenes described in order to highlight the theme of the plot and characterization of the main characters.

The novel is based on the story of the Song Dynasty uprising in the late Northern Song Dynasty. The whole story is mainly manifested by the praise of the heroes of the uprising and the depiction of the spirit of their struggle. Reflected in the back of the main are: the thirty-ninth back to the Xunyang House Song Jiangyin anti-poetry Liangshan Bo Dai Zong pass false letter, the seventy-first back to the loyalty hall stone tablets by astronomy Liangshan Park hero seat, seventy-seventh back Liangshan Park ten Face wandering Song Keming win-win hands, the first seven hundred and seven times Song Jiang victory over Ji Shanjun Zhu Wu broke the six flower array, the fifty-eighth back to the three mountains together to fight Qingzhou tigers tigers and so on. 
It is not difficult to see that these eyes are through the characters and places clever highlight the story content.

"Water Margin" to describe the character of the vivid description of the book successfully created a number of deeply rooted heroic characters, this feature can also be seen from the head back one or two. Such as the fourth back Zhao staff outside the re-repair Wenshuyuan Lu Zhishen hurriedly Wutai Mountain, the tenth back to the forest to teach the first snow-capped mountains Temple Lu Yu Hou fire burning material field, the seventeenth back to the monk two Longshan green beast two wins Baozhu Temple, the first hundred Zero five back Song Gongming summer heat soldiers Jun Daoguang back to the wind burned thieves Kou, the eighteenth back to the United States beard Gongzhi stability of the wing of the tiger Song Gong Ming explained Chao Tian Wang, the eighty-seventh Song Gongming war Youzhou call Yan Chong Chong Fan, Eighty-ninth return Song Gongming ruin success Su Qiu decrepit decrepit, the twenty-ninth back to the mercy of the Mengzhou Dawu Road, Wu Song drunk Jiang Men God forty-one return Song Jiangzhi take the army Zhang Shun live catch Huang Wenbing, Forty-fifth back to Yang Xiong drunk Pan Qiaoyun Shi Xiuzhi kill Pei like sea, the twenty-first back to the gods drunk playing Tang Niu song Jiang Yan killed Yan Piao, the sixth back, Kowloon pattern cut red pine forest Luzhi deep fire tile pot temple and so on.

To show the characteristics of the characters and their behavior in the back of the "Water Margin" is a part of the main body. In the above example, the expression effect is mostly positive or neutral, but also the description of the nature of the irony, such as the twenty-fifth back to the king of the poem sip Xi door prostitution medicine poison Wu Dalang, the twenty-fourth back to Wang Po bribery style Yun brother is not anger and so on. "Water Margin" depicts many distinctive characters, in the characterization of the characters; the biggest feature is that the author is good at putting the characters in the real historical environment, fastening the identity of the characters, experience and experience to describe their character. These images give the reader a fresh and vivid sense of lifelike historical situation vividly on paper. It is the success of these heroic image of the shape, is for this work has a glorious artistic life and influence.

In short, "Water Margin" as the earliest Zhanghui novel in Chinese history which is written in vernacular Chinese, its' chapter heading is of great value in literature, aesthetics and linguistics. It is also possible to study the content and the plot of the novel itself, and to analyze the use of the grammar of the novel itself. It can also combine the elements such as grammar, rhetoric, literature, aesthetics and national culture, and comprehensive cognitive connotation of chapter heading and antithesis.

\section{Acknowledgements}

Fund Project: Yulin High-level Talents Research Fund (12GK18).

\section{References}

[1] Shi Naian, Luo Guanzhong. Water Margin [M]. Changsha: Yuelu Library, 1988.

[2] Wang Xijie. Chinese rhetoric [M]. Beijing: Beijing Press, 1983.

[3] Yu Jingxiang. History of Chinese parallel prose [M]. Changchun: Jilin People's Publishing House, 2002.

[4] Zhu Chengping. Rhetoric of antithesis [M]. Changsha: Yuelu book society, 2003.

[5] Shi Lin. The source evolution and artistic implication of Chapter heading of Zhanghui novel [J]. Ming and Qing Dynasties novel research, 2004 (1) .22-31. 